\title{
VANISHING OF $\ell^{2}$-BETTI NUMBERS OF LOCALLY COMPACT GROUPS AS AN INVARIANT OF COARSE EQUIVALENCE
}

\author{
ROMAN SAUER AND MICHAEL SCHRÖDL
}

\begin{abstract}
We provide a proof that the vanishing of $\ell^{2}$-Betti numbers of unimodular locally compact second countable groups is an invariant of coarse equivalence. To this end, we define coarse $\ell^{2}$-cohomology for locally compact groups and show that it is isomorphic to continuous cohomology for unimodular groups and invariant under coarse equivalence.
\end{abstract}

\section{INTRODUCTION}

The insight that the vanishing of $\ell^{2}$-Betti numbers provides a quasiisometry invariant is due to Gromov (see [12, Chapter 8] for a statement without proof), and positive results around this insight have a long history. The most important contribution is by Pansu [18] whose work on asymptotic $\ell^{p}$-cohomology includes a proof that the vanishing of $\ell^{2}$-Betti numbers of discrete groups of type $F_{\infty}$, is a quasi-isometry invariant.

There is a growing interest in the metric geometry of locally compact groups [2,3]. We thus think it is important to have the quasi-isometry and coarse invariance of the vanishing of $\ell^{2}$-Betti numbers available in the greatest generality. Following Pansu's ideas and relying on more recent advances in the theory of $\ell^{2}$-Betti numbers, we provide a proof of the following result.

Theorem 1. Let $G$ and $H$ be unimodular locally compact second countable groups. If $G$ and $H$ are coarsely equivalent then the $n$-th $\ell^{2}$-Betti number of $G$ vanishes if and only the $n$-th $\ell^{2}$-Betti number of $H$ vanishes.

The coarse invariance for discrete groups was proven earlier in a paper of Mimura-Ozawa-Sako-Suzuki [16, Corollary 6.3].

Every locally compact, second countable group $G$ (hereafter abbreviated by lcsc) has a left-invariant proper continuous metric by a theorem of Struble [26]. As any two left-invariant proper continuous metrics on $G$ are coarsely equivalent, every lcsc group has a well defined coarse geometry. Further, any coarse equivalence between compactly generated lcsc groups

2010 Mathematics Subject Classification. Primary 20F65; Secondary 22D99.

Key words and phrases. Coarse geometry, locally compact groups, $\ell^{2}$-Betti numbers. 
is a quasi-isometry with respect to word metrics of compact symmetric generating sets and vice versa. In particular, a coarse equivalence between finitely generated discrete groups is a quasi-isometry. See [3, Chapter 4] for a systematic discussion of these notions.

To even state Theorem 1 in that generality, recent advances in the theory of $\ell^{2}$-Betti numbers were necessary. $\ell^{2}$-Betti numbers of discrete groups enjoy a long history but it was not until recently that $\ell^{2}$-Betti numbers were defined for arbitrary unimodular lcsc groups by Petersen [19], and a systematic theory analogous to the discrete case emerged [13, 19,20]. Earlier studies of $\ell^{2}$-Betti numbers of locally compact groups in specific cases can be found in [4, $\underline{6}, 10]$.

Previous results on coarse invariance. Pansu [18] introduced asymptotic $\ell^{p}$-cohomology of discrete groups and proved its invariance under quasiisometries. If a group $\Gamma$ is of type $F_{\infty}$, then the $\ell^{p}$-cohomology of $\Gamma$ coincides with its asymptotic $\ell^{p}$-cohomology [18, Théorème 1]. The geometric explanation for the appearance of the type $F_{\infty}$ condition is that the finitedimensional skeleta of the universal covering of a classifying space of finite type are uniformly contractible. As an immediate consequence of Pansu's result, the vanishing of $\ell^{2}$-Betti numbers is a quasi-isometry invariant among discrete groups of type $F_{\infty}$. The same arguments work for totally disconnected groups admitting a topological model of finite type [23].

Elek [7] investigated the relation between $\ell^{p}$-cohomology of discrete groups and Roe's coarse cohomology and proved similar results. Another independent treatment is due to Fan [8]. Genton [11] elaborated upon Pansu's methods in the case of metric measure spaces.

Oguni [17] generalised the quasi-isometry invariance of the vanishing of $\ell^{2}$-Betti numbers from discrete groups of type $F_{\infty}$ to discrete groups whose cohomology with coefficients in the group von Neumann algebra satisfies a certain technical condition. A similar technical condition appears in the proof of quasi-isometry invariance of Novikov-Shubin invariants of amenable groups [25], and it is unclear how much this condition differs from the type $F_{\infty}$-condition. Oguni's groupoid approach is inspired by [9, 25] and quite different from the approaches by Elek, Fan, and Pansu.

The coarse invariance of vanishing of $\ell^{2}$-Betti numbers for discrete groups was shown by Mimura-Ozawa-Sako-Suzuki [16, Corollary 6.3]. Li [14] recently reproved this using groupoid techniques as a consequence of more general cohomological coarse invariance results. 
Structure of the paper. We review the necessary basics of $\ell^{2}$-Betti numbers and continuous cohomology in Section 2. In Section 3 we define coarse $\ell^{2}$ cohomology for lcsc groups and show that it is isomorphic to continuous cohomology. In Section 4 we conclude the proof of Theorem 1 and discuss what fails for non-unimodular groups.

\section{Continuous Cohomology and $\ell^{2}$-Betti numbers of LCSC GROUPS}

Let $G$ be a unimodular lcsc group with Haar measure $\mu$. Let $X$ be a locally compact second countable space with Radon measure $\nu$. Let $E$ be a Fréchet space.

The space $C(X, E)$ of continuous functions from $X$ to $E$ becomes a Fréchet space when endowed with the topology of compact convergence. Let $L_{l o c}^{2}(X, E)$ be the space of equivalence classes of measurable maps $f: X \rightarrow E$ up to $\nu$-null sets such that $\left.|| f\right|_{K} \|_{E}$ is square-integrable for every compact subset $K \subset X$. The $L^{2}$-norm of the function $\left\|\left.f\right|_{K}\right\|_{E}$ defines a semi-norm $p_{K}$ on $L_{l o c}^{2}(X, E)$. The family of semi-norms $p_{K}, K \subset E$, turns $L_{l o c}^{2}(X, E)$ into a Fréchet space.

We call a Fréchet space $E$ with a continuous (i.e. $G \rightarrow E, g \mapsto g v$, is continuous for every $v \in E$ ) linear $G$-action a $G$-module. A continuous linear $G$-equivariant map between $G$-modules is a homomorphism of $G$-modules. If $E$ is a $G$-module and $G$ acts continuously and $\nu$-preserving on $X$ then $C(X, E)$ and $L_{l o c}^{2}(X, E)$ become $G$-modules via $(g \cdot f)(x)=g f\left(g^{-1} x\right)$ for $x \in X$ and $g \in G$ [1, Proposition 3.1.1]. The usual homogeneous coboundary map

$$
d^{n-1} f\left(g_{0}, \ldots, g_{n}\right)=\sum_{i=0}^{n}(-1)^{i} f\left(g_{0}, \ldots, \widehat{g}_{i}, \ldots, g_{n}\right)
$$

defines cochain complexes $C\left(G^{*+1}, E\right)$ and $L_{l o c}^{2}\left(G^{*+1}, E\right)$ of $G$-modules (cf. [1, Proposition 3.2.1]). Here we take the diagonal $G$-action on $G^{*+1}$. We recall the following definition.

Definition 2. The (continuous) cohomology of $G$ with coefficients in $E$ is the cohomology

$$
H^{n}(G, E)=H^{n}\left(C\left(G^{*+1}, E\right)^{G}\right)
$$

of the $G$-invariants of $C\left(G^{*+1}, E\right)$. The reduced (continuous) cohomology $\underline{H}^{*}(G, E)$ is a quotient of $H^{*}(G, E)$ obtained by taking the quotient with the closure of im $d^{*-1}$ instead of im $d^{*-1}$. 
We have an obvious inclusion

$$
I^{*}: C\left(G^{*+1}, E\right) \rightarrow L_{l o c}^{2}\left(G^{*+1}, E\right) .
$$

The maps $I^{*}$ form a cochain map of $G$-modules. Taking a positive function $\chi \in C_{c}(G)$ there is a cochain map $R^{*}: L_{l o c}^{2}\left(G^{*+1}, E\right) \rightarrow C\left(G^{*+1}, E\right)$ of $G$-modules

$$
\left(R^{n} f\right)\left(g_{0}, \ldots, g_{n}\right)=\int_{G^{n+1}} f\left(h_{0}, \ldots, h_{n}\right) \chi\left(g_{0}^{-1} h_{0}\right) \cdot \ldots \cdot \chi\left(g_{n}^{-1} h_{n}\right) d \mu\left(h_{0}, \ldots, h_{n}\right)
$$

such that $I^{*} \circ R^{*}$ and $R^{*} \circ I^{*}$ are homotopic (as cochain maps of $G$-modules) to the identity [1, Proposition 4.8]. So we have the following useful fact:

Theorem 3. The cochain map $I^{*}$ in (2) induces isomorphisms in cohomology and in reduced cohomology.

Next we turn to the case where the coefficient module $E=L^{2}(G)$ is the regular representation, relevant for the definition of $\ell^{2}$-Betti numbers.

Let $L(G)$ be the von Neumann algebra of $G$; the Haar measure $\mu$ defines a semifinite $\operatorname{trace} \operatorname{tr}_{\mu}$ on $L(G)$. There are a natural left $G$-action and a natural right $L(G)$-action on $L^{2}(G)$, and the two actions commute. Hence also the $G$ actions on $C\left(G^{*+1}, L^{2}(G)\right)$ and $L_{l o c}^{2}\left(G^{*+1}, L^{2}(G)\right)$ considered previously and the $L(G)$-actions induced from the right $L(G)$-action on $L^{2}(G)$ commute. So the (reduced and non-reduced) continuous cohomology of $G$ with coefficients in $L^{2}(G)$ is naturally a $L(G)$-modul 1 . Obviously, the cochain map $I^{*}$ above is compatible with the $L(G)$-module structures. The groups $H^{*}\left(G, L^{2}(G)\right)$ are called the (continuous) $\ell^{2}$-cohomology of $G$. Similarly for the reduced cohomology.

Petersen [19] extended Lück's dimension function from finite von Neumann algebras to semifinite von Neumann algebras. The dimension function $\operatorname{dim}_{\mu}$ with respect to $(G, \mu)$ is a non-trivial dimension for (algebraic) right $L(G)$-modules that is additive for short exact sequences of $L(G)$-modules. It scales as $\operatorname{dim}_{c \mu}=c^{-1} \operatorname{dim}_{\mu}$ for $c>0$. The fact that a $L(G)$-module has dimension zero can be expressed without referring to the trace: it is an algebraic fact. The following criterion was shown by the first author for finite von Neumann algebras [24, Theorem 2.4]; it was extended to the semifinite case by Petersen [19, Lemma B.27].

Theorem 4. An $L(G)$-module $M$ satisfies $\operatorname{dim}_{\mu}(M)=0$ if and only if for every $x \in M$ there is an increasing sequence $\left(p_{i}\right)$ of projections in $L(G)$ with $\sup p_{i}=1$ such that $x p_{i}=0$ for every $i \in \mathbb{N}$.

\footnotetext{
${ }^{1}$ When talking about $L(G)$-modules we mean the algebraic module structure and ignore topologies.
} 
Definition 5. The $n$-th $\ell^{2}$-Betti number of $G$ is the $L(G)$-dimension of its reduced continuous cohomology with coefficients in $L^{2}(G)$, i.e.

$$
\beta_{(2)}^{n}(G):=\operatorname{dim}_{\mu} \underline{H}^{n}\left(G, L^{2}(G)\right) \in[0, \infty] .
$$

Remark 6. Equivalently, the $n$-th $\ell^{2}$-Betti number can be defined as the $L(G)$-dimension of the non-reduced cohomology $H^{n}\left(G, L^{2}(G)\right)$. This is a non-trivial fact (see [13, Theorem A]). For discrete $G$, our definition coincides with Lück's definition in [15]. Again, this is non-trivial and shown in [21, Theorem 2.2].

The following lemma was observed in [19, Proposition 3.8]. Since it is a direct consequence of Theorem 4 we present the argument.

Lemma 7. $\beta_{(2)}^{n}(G)=0 \Leftrightarrow \underline{H}^{n}\left(G, L^{2}(G)\right)=0$.

Proof. Let $\beta_{(2)}^{n}(G)=0$. Let $f: G^{n+1} \rightarrow L^{2}(G)$ be a cocycle representing a cohomology class $[f]$ in $\underline{H}^{n}\left(G, L^{2}(G)\right)$. By Theorem 4 there is an increasing sequence of projections $p_{j} \in L(G)$ whose supremum is 1 such that each $f p_{j}$ is a coboundary $d^{n-1} b_{j}$. It is clear that $f p_{j}=d^{n-1} b_{j}$ converges to $f$ in the topology of $C\left(G^{n+1}, L^{2}(G)\right)$, thus $[f]=0$.

\section{Coarse equivalence and coArse $\ell^{2}$-COHomology}

Let $G$ be a lcsc group. We fix a left-invariant proper continuous metric $d$ on $G$. Let $\mu$ be a Haar measure on $G$. Let $\mu_{n}$ be the $n$-fold product measure of $\mu$ on $G^{n}$.

For every $R>0$ and $n \in \mathbb{N}_{0}$ we consider the closed subset

$$
G_{R}^{n}:=\left\{\left(g_{0}, \ldots, g_{n-1}\right) \in G^{n} \mid d\left(g_{i}, g_{j}\right) \leq R \text { for all } 0 \leq i, j \leq n-1\right\}
$$

and a family of semi-norms for measurable maps $\alpha: G^{n+1} \rightarrow \mathbb{C}$ defined by

$$
\|\alpha\|_{R}^{2}=\int_{G_{R}^{n+1}}\left|\alpha\left(g_{0}, \ldots, g_{n}\right)\right|^{2} d \mu_{n+1} \in[0, \infty] .
$$

Let $C X_{(2)}^{n}(G)$ be the space of equivalence classes (up to $\mu_{n+1}$-null sets) of measurable maps $\alpha: G^{n+1} \rightarrow \mathbb{C}$ such that $\|\alpha\|_{R}<\infty$ for every $R>$ 0 . The semi-norms $\left\|_{-}\right\|_{R}, R>0$, turn $C X_{(2)}^{n}(G)$ into a Fréchet space. It is straightforward to verify that the homogeneous differential (11) yields a well-defined, continuous homomorphism $C X_{(2)}^{n}(G) \rightarrow C X_{(2)}^{n+1}(G)$ (cf. [11, Proposition 2.3.3]). Thus we obtain a cochain complex of Fréchet spaces.

Definition 8. The coarse $\ell^{2}$-cohomology of $G$ is defined as

$$
H X_{(2)}^{n}(G)=H^{n}\left(C X_{(2)}^{*}(G)\right)
$$


By taking the quotients by the closure of the differentials, one defines similarly the reduced coarse $\ell^{2}$-cohomology $\underline{H} X_{(2)}^{n}(G)$.

Remark 9. The previous definition is the continuous analog of Elek's definition [7, Definition 1.3] in the discrete case (Elek gives credits to Roe [22]). It is very much related to Pansu's asymptotic $\ell^{2}$-cohomology [18], which was considered in the generality of metric measure spaces by Genton [11]. The difference of our definition to the one in Genton [11] is as follows: $C X_{(2)}^{*}(G)$ is an inverse limit of spaces $L^{2}\left(G_{R}^{*+1}\right)$. Unlike us, Genton takes first the cohomology of $L^{2}\left(G_{R}^{*+1}\right)$ and then the inverse limit. Under some uniform contractibility assumptions the two definitions coincide but likely not in general.

Theorem 10. Let $G$ be a unimodular lcsc group. For every $n \geq 0$, the $n$-th continuous cohomology of $G$ with coefficients in the left regular representation $L^{2}(G)$ is isomorphic to the $n$-th coarse $\ell^{2}$-cohomology of $G$, and likewise for reduced cohomology.

Proof. We have the obvious embedding

$$
L_{l o c}^{2}\left(G^{n+1}, L^{2}(G)\right) \subset L_{l o c}^{2}\left(G^{n+1}, L_{l o c}^{2}(G)\right)
$$

and the exponential law (see [1, Lemme 1.4] for a proof but beware of the typo in the statement)

$$
L_{l o c}^{2}\left(G^{n+1}, L_{l o c}^{2}(G)\right) \cong L_{l o c}^{2}\left(G^{n+1} \times G\right) .
$$

Thus an element in $L_{l o c}^{2}\left(G^{n+1}, L^{2}(G)\right)^{G}$ is represented by a measurable complex function in $(n+2)$-variables. For $\alpha \in L_{l o c}^{2}\left(G^{n+1}, L^{2}(G)\right)^{G}$ we define $\mu_{n+2}$-almost everywhere

$$
F^{n}(\alpha)\left(x_{0}, \ldots, x_{n}, x\right)=\alpha\left(x^{-1} x_{0}, \ldots, x^{-1} x_{n}\right)(x) .
$$

The measurable function $F^{n}(\alpha)$ is invariant by translation in the $(n+2)$ th variable. By Fubini's theorem we may regard $F^{n}(\alpha)$ as a measurable function $E^{n}(\alpha): G^{n+1} \rightarrow \mathbb{C}$ in the first $(n+1)$-variables. We may think of $E^{n}(\alpha)$ as an evaluation of $\alpha$ at $e$. Let $B(R)$ denote the $R$-ball around $e \in G$. Next we show that $\left\|E^{n}(\alpha)\right\|_{R}<\infty$ for every $R>0$, thus $E^{n}(\alpha) \in C X_{(2)}^{n}(G)$.

Since $\alpha \in L_{l o c}^{2}\left(G^{n+1}, L^{2}(G)\right)^{G}$ we have

$$
\begin{aligned}
\infty & >\int_{B(2 R)^{n+1}} \int_{G}\left|\alpha\left(x_{0}, x_{1}, \ldots, x_{n}\right)(x)\right|^{2} d \mu d \mu_{n+1} \\
& =\int_{B(2 R)^{n+1}} \int_{G}\left|\alpha\left(x, x x_{0}^{-1} x_{1}, \ldots, x x_{0}^{-1} x_{n}\right)\left(x_{0}\right)\right|^{2} d \mu d \mu_{n+1} .
\end{aligned}
$$


The map

$$
m: G^{n+2} \rightarrow G^{n+2},\left(x_{0}, \ldots, x_{n}, x\right) \mapsto\left(x, x x_{0}^{-1} x_{1}, \ldots, x x_{0}^{-1} x_{n}, x_{0}\right)
$$

is measure preserving since it is the composition of taking inverses in the last coordinate, left multiplication by $x x_{0}^{-1}$, conjugation by $x$ and taking inverses in the last coordinate. Note that this requires unimodularity. Further, we have

$$
m\left(G_{R}^{n+1} \times B(R)\right) \subset B(2 R)^{n+1} \times G .
$$

This implies the first inequality below. The first equality follows from the fact that $\left(x_{0}, \ldots, x_{n}, x\right) \mapsto\left(x^{-1} x_{0}, \ldots, x^{-1} x_{n}, x\right)$ is a measure preserving measurable automorphism of $G_{R}^{n+1} \times B(R)$.

$$
\begin{aligned}
\int_{B(2 R)^{n+1}} \int_{G}\left|\alpha\left(x, x x_{0}^{-1} x_{1}, \ldots, x x_{0}^{-1} x_{n}\right)\left(x_{0}\right)\right|^{2} d \mu d \mu_{n+1} \\
\quad \geq \int_{G_{R}^{n+1}} \int_{B(R)}\left|\alpha\left(x_{0}, \ldots, x_{n}\right)(x)\right|^{2} d \mu d \mu_{n+1} \\
=\int_{G_{R}^{n+1}} \int_{B(R)}\left|\alpha\left(x^{-1} x_{0}, \ldots, x^{-1} x_{n}\right)(x)\right|^{2} d \mu d \mu_{n+1} \\
=\mu(B(R))\left\|E^{n}(\alpha)\right\|_{R} .
\end{aligned}
$$

Hence $\left\|E^{n}(\alpha)\right\|_{R}$ is finite for every $R>0$. That

$$
E^{*}: L_{l o c}^{2}\left(G^{*+1}, L^{2}(G)\right)^{G} \rightarrow C X_{(2)}^{*}(G)
$$

defines a cochain map is obvious. The above computation also implies that $E^{*}$ is continuous with respect to the Fréchet topologies.

Given $\beta \in C X_{(2)}^{n}(G)$ we define

$$
M^{n}(\beta)\left(g_{0}, \ldots, g_{n}\right)(g)=\beta\left(g^{-1} g_{0}, \ldots, g^{-1} g_{n}\right)
$$

for $\mu_{n+2}$-almost every $\left(g_{0}, \ldots, g_{n}, g\right)$. The function $M^{n}(\beta)$ defines an element in $L_{l o c}^{2}\left(G^{n+1}, L^{2}(G)\right)^{G}$. The $G$-invariance of $M^{n}(\beta)$ is obvious. We have to show that $\left\|\left.M^{n}(\beta)\right|_{B(R)^{n+1}}\right\|$ is square-integrable for every $R>0$. This follows from the following computations which is based on the arguments above in reversed order.

$$
\begin{aligned}
\mu(B(R)) \int_{G_{2 R}^{n+1}}\left|\beta\left(g_{0}, \ldots, g_{n}\right)\right|^{2} d \mu_{n+1} \\
\quad=\int_{G_{2 R}^{n+1}} \int_{B(R)}\left|\beta\left(g_{0}, \ldots, g_{n}\right)\right|^{2} d \mu d \mu_{n+1} \\
\geq \int_{B(R)^{n+1}} \int_{G}\left|\beta\left(g^{-1} g_{0}, \ldots, g^{-1} g_{n}\right)\right| d \mu d \mu_{n+1}
\end{aligned}
$$


Obviously, $M^{*}$ is a chain map. Continuity follows from the previous computation. It is clear that $M^{*}$ and $E^{*}$ are mutual inverses. Using Theorem 3 , this concludes the proof.

\section{CoArse invariance}

We recall the notion of coarse equivalence. A map $f:\left(X, d_{X}\right) \rightarrow\left(Y, d_{Y}\right)$ between metric spaces is coarse Lipschitz if there is a non-decreasing function $a:[0, \infty) \rightarrow[0, \infty)$ with $\lim _{t \rightarrow \infty} a(t)=\infty$ such that

$$
d_{Y}\left(f(x), f\left(x^{\prime}\right)\right) \leq a\left(d\left(x, x^{\prime}\right)\right)
$$

for all $x, x^{\prime} \in X$. We say that two such maps $f, g$ are close if

$$
\sup _{x \in X} d_{Y}(f(x), g(x))<\infty .
$$

A coarse Lipschitz map $f: X \rightarrow Y$ is a coarse equivalence if there is a coarse Lipschitz map $g: Y \rightarrow X$ such that $f g$ and $g f$ are close to the identity. We say $g$ is a coarse inverse of $f$.

Lemma 11. Coarsely equivalent lcsc groups are measurably coarse equivalent, i.e. if $G$ and $H$ are coarse equivalent lcsc groups then there are measurable coarse Lipschitz maps $f: G \rightarrow H$ and $g: H \rightarrow G$ such that $f g$ and gf are close to the identity.

Proof. We choose left-invariant continuous proper metrics $d_{G}$ and $d_{H}$ on $G$ and $H$, respectively. Let $f: G \rightarrow H$ be a coarse Lipschitz map with $d_{H}\left(f(x), f\left(x^{\prime}\right)\right) \leq a\left(d_{G}\left(x, x^{\prime}\right)\right)$. Let $t>0$. We pick a countable measurable partition $\mathcal{U}$ of $G$ whose elements have diameter $\leq t$ and choose an element $x_{U} \in U$ for every $U \in \mathcal{U}$.

By setting $\tilde{f}(x)=f\left(x_{U}\right)$ for $x \in U$ we obtain a coarse Lipschitz map $\tilde{f}: G \rightarrow H$ which satisfies $d\left(\tilde{f}(x), \tilde{f}\left(x^{\prime}\right)\right) \leq a\left(d\left(x, x^{\prime}\right)+2 t\right)$ and is close to $f$ with $d(\tilde{f}(x), f(x)) \leq a(2 t)$. Analogously, we construct a measurable coarse Lipschitz map $\tilde{g}$, constructed from a coarse Lipschitz map $g: H \rightarrow G$ which is a coarse inverse to $f$. It is obvious that $\tilde{g}$ is a coarse inverse to $\tilde{f}$.

Theorem 12. Coarsely equivalent lcsc groups have isomorphic reduced and non-reduced coarse $\ell^{2}$-cohomology groups.

Proof. Let $G$ and $H$ lcsc groups with Haar measures $\mu$ and $\nu$, respectively. Let $f: G \rightarrow H$ be a coarse equivalence with coarse inverse $g$. Because of lemma 11 we can further assume that $f$ and $g$ are measurable. We define a $\operatorname{map} \chi: G \times G \rightarrow \mathbb{R}$ by

$$
\chi(x, y)=\frac{\mathbb{1}_{B_{x}(c)}(y)}{\mu(B(c))}
$$


where we choose $c$ such that $\mu(B(c)) \geq 1$. Then $\chi$ is a measurable function with $\chi(x, y)=\chi(y, x)$ and $\int_{G} \chi(x, y) d \mu(y)=1$. We use the following notation:

$\chi: G^{n+1} \times G^{n+1} \rightarrow \mathbb{R}, \quad \chi\left(\left(x_{0}, \ldots, x_{n}\right),\left(y_{0}, \ldots, y_{n}\right)\right)=\chi\left(x_{0}, y_{0}\right) \cdot \ldots \cdot \chi\left(x_{n}, y_{n}\right)$.

Analogously, we define $\chi^{\prime}: H^{n+1} \times H^{n+1} \rightarrow \mathbb{R}$ with some radius $c^{\prime}$. Now we can define the maps $f^{*}: H X_{(2)}^{*}(H) \rightarrow H X_{(2)}^{*}(G)$ and $g^{*}: H X_{(2)}^{*}(G) \rightarrow$ $H X_{(2)}^{*}(H)$ as follows where we use $x_{i}$ for elements in $G$ and $y_{i}$ for elements of $H$ :

$$
\begin{aligned}
f^{*} \alpha\left(x_{0}, \ldots, x_{n}\right) & =\int_{H^{n+1}} \alpha\left(y_{0}, \ldots, y_{n}\right) \chi^{\prime}\left(\left(f\left(x_{0}\right), \ldots, f\left(x_{n}\right)\right),\left(y_{0}, \ldots, y_{n}\right)\right) d \nu_{n+1} \\
g^{*} \beta\left(y_{0}, \ldots, y_{n}\right) & =\int_{G^{n+1}} \beta\left(x_{0}, \ldots, x_{n}\right) \chi\left(\left(g\left(y_{0}\right), \ldots, g\left(y_{n}\right)\right),\left(x_{0}, \ldots, x_{n}\right)\right) d \mu_{n+1} .
\end{aligned}
$$

The idea of averaging over a function like $\chi$ goes back to Pansu; it is necessary in our context since the maps $f$ and $g$ do not preserve the measure classes, in general.

First of all, we check that these are well-defined continuous cochain maps.

$$
\begin{aligned}
& >\|\alpha\|_{a(R)+c^{\prime}}^{2}=\int_{H^{n+1}}\left|\alpha\left(y_{0}, \ldots, y_{n}\right)\right|^{2} \cdot \mathbb{1}_{H_{a(R)+c^{\prime}}^{n}} d \nu_{n+1} \\
\geq & \int_{H^{n+1}}\left|\alpha\left(y_{0}, \ldots, y_{n}\right)\right|^{2} \int_{G_{R}^{n+1}} \chi^{\prime}\left(\left(f\left(x_{0}\right), \ldots, f\left(x_{n}\right)\right),\left(y_{0}, \ldots, y_{n}\right)\right) d \mu_{n+1} d \nu_{n+1} \\
= & \int_{G_{R}^{n+1}} \int_{H^{n+1}}\left|\alpha\left(y_{0}, \ldots, y_{n}\right)\right|^{2} \chi^{\prime}\left(\left(f\left(x_{0}\right), \ldots, f\left(x_{n}\right)\right),\left(y_{0}, \ldots, y_{n}\right)\right) d \nu_{n+1} d \mu_{n+1} \\
\geq & \int_{G_{R}^{n+1}}\left|\int_{H^{n+1}} \alpha\left(y_{0}, \ldots, y_{n}\right) \chi^{\prime}\left(\left(f\left(x_{0}\right), \ldots, f\left(x_{n}\right)\right),\left(y_{0}, \ldots, y_{n}\right)\right) d \nu_{n+1}\right|^{2} d \mu_{n+1} \\
= & \int_{G_{R}^{n+1}}\left|f^{n} \alpha\left(x_{0}, \ldots, x_{n}\right)\right|^{2} d \mu_{n+1}=\left\|f^{n} \alpha\right\|_{R}^{2}
\end{aligned}
$$

It is a direct computation that $d^{n} \circ f^{n}=f^{n+1} \circ d^{n}$.

It remains to show that there is a cochain homotopy $h: C X_{(2)}^{*}(H) \rightarrow$ $C X_{(2)}^{*-1}(H)$ such that $\mathrm{Id}-g^{*} f^{*}=h d+d h$. We define $h_{i}^{n+1}: C X_{(2)}^{n+1}(H) \rightarrow$ $C X_{(2)}^{n}(H)$ by

$$
\begin{aligned}
& h_{i}^{n+1} \alpha\left(y_{0}, \ldots, y_{n}\right) \\
= & \int_{H^{n+1}} \alpha\left(\tilde{y}_{0}, \ldots, \tilde{y}_{i}, y_{i}, \ldots, y_{n}\right) \chi^{\prime}\left(\left(y_{0}, \ldots, y_{n}\right),\left(\tilde{y}_{0}, \ldots, \tilde{y}_{n}\right)\right) d \nu_{n+1}(\tilde{y})
\end{aligned}
$$

and set

$$
h^{n+1}=\sum_{i=0}^{n}(-1)^{i} h_{i}^{n+1} .
$$

That $h^{*}$ is well-defined is a similar consideration as to show that $f^{*}$ and $g^{*}$ are well-defined. Now let us denote the i-th term of the coboundary map 
by $d_{i}^{n}$, i.e. $d_{i}^{n} \alpha\left(y_{0}, \ldots, y_{n+1}\right)=\alpha\left(y_{0}, \ldots, \widehat{y}_{i}, \ldots, y_{n+1}\right)$. It is straightforward to verify that we have the following relations:

$$
\begin{array}{rlrl}
h_{n}^{n+1} \circ d_{n+1}^{n} & =g^{n} \circ f^{n}, & \\
h_{0}^{n+1} \circ d_{0}^{n} & =\operatorname{Id}_{C X_{(2)}^{n}(H)}, & & \\
h_{j}^{n+1} \circ d_{i}^{n} & =d_{i}^{n-1} \circ h_{j-1}^{n} & & \text { for } 1 \leq j \leq n \text { and } i \leq j, \\
h_{j}^{n+1} \circ d_{i}^{n} & =d_{i-1}^{n-1} \circ h_{j}^{n} & & \text { for } 1 \leq i \leq n \text { and } i>j .
\end{array}
$$

We get $h^{n+1} d^{n}+d^{n-1} h^{n}=\operatorname{Id}_{C X_{(2)}^{n}(H)}-g^{n} \circ f^{n}$. The same construction applies to $f^{*} g^{*}$ which completes the proof.

Proof of Theorem 11. Let $G$ and $H$ be unimodular lcsc groups. Let $G$ and $H$ be coarsely equivalent. Then we have the following equivalences:

$$
\begin{aligned}
\beta_{(2)}^{2}(G)=0 & \Leftrightarrow \underline{H}^{n}\left(G, L^{2}(G)\right)=0 & & \text { (Lemma 7) } \\
& \Leftrightarrow \underline{H} X_{(2)}^{n}(G)=0 & & (\text { Theorem 10) } \\
& \Leftrightarrow \underline{H} X_{(2)}^{n}(H)=0 & & \text { (Theorem 12) }
\end{aligned}
$$

Going the same steps backwards for the group $H$ finishes the proof.

Remark 13. Since the Borel subgroup $B<\mathrm{SL}_{2}(\mathbb{R})$ of upper triangular matrices is cocompact, the solvable Lie groups $B$ and $\mathrm{SL}_{2}(\mathbb{R})$ are quasiisometric. So $B$ belongs to the class of amenable hyperbolic lcsc groups of which a systematic study was undertaken in [2].

The group $B$ is not unimodular and thus its $\ell^{2}$-Betti number are not defined. Nevertheless, one may ask what exactly breaks down in the proof above which can be formulated to a large part without the notion of $\ell^{2}$-Betti numbers. By a result of Delorme [5, Corollaire V.3], we have $\left.\underline{H}^{1}\left(B, L^{2}(B)\right)\right)=$ 0 . Since Theorem 12 does not require unimodularity, we have $\underline{H} X_{(2)}^{1}(B) \cong$ $\underline{H} X_{(2)}^{n}\left(\mathrm{SL}_{2}(\mathbb{R})\right) \neq 0$ since $\beta_{(2)}^{1}\left(\mathrm{SL}_{2}(\mathbb{R})\right) \neq 0$. So it is Theorem 10 that fails for the non-unimodular group $B$.

Acknowledgements. We acknowledge support by the German Science Foundation via the Research Training Group 2229.

\section{REFERENCES}

[1] P. Blanc, Sur la cohomologie continue des groupes localement compacts, Ann. Sci. École Norm. Sup. (4) 12 (1979), 137-168.

[2] P.-E. Caprace et al., Amenable hyperbolic groups, J. Eur. Math. Soc. (JEMS) 17 (2015), 2903-2947. 
[3] Y. Cornulier and P. de la Harpe, Metric geometry of locally compact groups, EMS Tracts in Mathematics, 25, European Mathematical Society (EMS), Zürich, 2016.

[4] M. W. Davis et al., Weighted $L^{2}$-cohomology of Coxeter groups, Geom. Topol. 11 (2007), 47-138.

[5] P. Delorme, 1-cohomologie des représentations unitaires des groupes de Lie semi-simples et résolubles. Produits tensoriels continus de représentations, Bull. Soc. Math. France 105 (1977), 281-336.

[6] J. Dymara, Thin buildings, Geom. Topol. 10 (2006), 667-694.

[7] G. Elek, Coarse cohomology and $l_{p}$-cohomology, K-Theory 13 (1998), $1-22$.

[8] P. T. Fan, Coarse $l_{p}$-geometic invariants, ProQuest LLC, Ann Arbor, MI, 1993.

[9] D. Gaboriau, Invariants $l^{2}$ de relations d'équivalence et de groupes, Publ. Math. Inst. Hautes Études Sci. 95 (2002), 93-150.

[10] D. Gaboriau, Invariant percolation and harmonic Dirichlet functions, Geom. Funct. Anal. 15 (2005), 1004-1051.

[11] L. Genton, Scaled Alexander-Spanier Cohomology and $L^{q, p}$ Cohomology for Metric Spaces, EPFL, Lausanne, Thèse no 6330, (2014).

[12] M. Gromov, Asymptotic invariants of infinite groups, in Geometric group theory, Vol. 2 (Sussex, 1991), 1-295, London Math. Soc. Lecture Note Ser., 182, Cambridge Univ. Press, Cambridge.

[13] D. Kyed, H. D. Petersen and S. Vaes, $L^{2}$-Betti numbers of locally compact groups and their cross section equivalence relations, Trans. Amer. Math. Soc. 367 (2015), 4917-4956.

[14] X. Li, Dynamic characterizations of quasi-isometry, and applications to cohomology, arXiv:1604.07375.

[15] W. Lück, Dimension theory of arbitrary modules over finite von Neumann algebras and $L^{2}$-Betti numbers, I. Foundations, J. Reine Angew. Math. 495 (1998), 135-162.

[16] M. Mimura et al., Group approximation in Cayley topology and coarse geometry, III: Geometric property (T), Algebr. Geom. Topol. 15 (2015), 1067-1091.

[17] S. Oguni, $L^{2}$-Invariants of discrete groups under coarse equivalence and $L^{2}$-invariants of cocompact etale groupoids, preprint (2010).

[18] P. Pansu, Cohomologie $L^{p}$ : invariance sous quasiisométries, preprint (1995). 
[19] H. D. Petersen, $L^{2}$-Betti numbers of locally compact groups, C. R. Math. Acad. Sci. Paris 351 (2013), 339-342.

[20] H.D. Petersen, R. Sauer and A. Thom, $L^{2}$-Betti numbers of totally disconnected groups and their approximation by Betti numbers of lattices, arXiv:1612.04559. To appear in Journal of Topology.

[21] J. Peterson and A. Thom, Group cocycles and the ring of affiliated operators, Invent. Math. 185 (2011), 561-592.

[22] J. Roe, Coarse cohomology and index theory on complete Riemannian manifolds, Mem. Amer. Math. Soc. 104 (1993), 1-22.

[23] R. Sauer, $\ell^{2}$-Betti numbers of discrete and non-discrete groups, in: New directions in locally compact groups, London Mathematical Society Lecture Note Series 447, Cambridge University Press, 2018, 205-226.

[24] R. Sauer, $L^{2}$-Betti numbers of discrete measured groupoids, Internat. J. Algebra Comput. 15 (2005), 1169-1188.

[25] R. Sauer, Homological invariants and quasi-isometry, Geom. Funct. Anal. 16 (2006), 476-515.

[26] R. A. Struble, Metrics in locally compact groups, Compositio Math. 28 (1974), 217-222.

Institute for Algebra and Geometry, Karlsruhe Institute of Technology, Englerstr. 2, 76128 Karlsruhe, Germany

E-mail address: roman.sauer@kit.edu

Institute for Algebra and Geometry, Karlsruhe Institute of Technology, Englerstr. 2, 76128 Karlsruhe, Germany

E-mail address: michael.schroedl@kit.edu 